\title{
ENSEMBLES OF MULTI-INSTANCE NEURAL NETWORKS
}

\author{
Min-Ling Zhang, Zhi-Hua Zhou \\ National Laboratory for Novel Software Technology, Nanjing University, \\ Nanjing 210093, China \\ zhangml@lamda.nju.edu.cn zhouzh@nju.edu.cn
}

Abstract: $\quad$ Recently, multi-instance classification algorithm BP-MIP and multi-instance regression algorithm BP-MIR both based on neural networks have been proposed. In this paper, neural network ensemble techniques are introduced to solve multi-instance learning problems, where BP-MIP ensemble and BP-MIR ensemble are constructed respectively. Experiments on benchmark and artificial data sets show that ensembles of multi-instance neural networks are superior to single multi-instance neural networks in solving multi-instance problems.

Key words: machine learning; multi-instance learning; neural networks; neural network ensemble.

\section{INTRODUCTION}

The notion of multi-instance learning was proposed by Dietterich et al. [2] in their investigation of drug activity prediction. In multi-instance learning, the training set is composed of many bags each containing many instances. If a bag contains at least one positive instance then it is labeled as a positive bag. Otherwise it is labeled as a negative bag. The labels of the training bags are known, but those of the training instances are unknown. The task is to learn something from the training set for correctly labeling unseen bags. Due to its unique characteristics and extensive applicability, multi-instance learning has been regarded as a new learning framework parallel to supervised learning, unsupervised learning, and reinforcement learning [4].

Recently, neural network based multi-instance classification algorithm BP-MIP [9] and regression algorithm BP-MIR [7] have been proposed, both 
of which are derived from the popular BP algorithm [6] with a global error function defined at the level of bags instead of at the level of instances. Considering that ensemble learning has been used to significantly improve the generalization ability of several multi-instance learners [10], this paper proposes to build ensembles of multi-instance neural networks to solve multi-instance problems, where BP-MIP ensemble and BP-MIR ensemble are constructed respectively. Experiments on benchmark and artificial data sets show that ensembles of multi-instance neural networks are superior to single multi-instance neural networks in solving multi-instance problems.

The rest of this paper is organized as follows. Section 2 proposes to build ensemble of BP-MIP. Section 3 proposes to build ensemble of BP-MIR. Finally, Section 4 concludes and indicates several issues for future work. Due to the page limitation, for more information about multi-instance learning, BP-MIP and BP-MIR, please refer to the literatures [7] and [8].

\section{BP-MIP ENSEMBLE}

The Musk data is the only real-world benchmark test data for multiinstance learning at present. There are two data sets, both of which are publicly available from UCI Machine Learning Repository. Characteristics of those two data sets are summarized in Table 1.

Table 1. Some characteristics of the Musk data

\begin{tabular}{lcc}
\hline Data set & Musk1 & Musk2 \\
\hline Dimensionality & 166 & 166 \\
Number of bags & 92 & 102 \\
Number of positive bags & 47 & 39 \\
Number of negative bags & 45 & 63 \\
Number of instances & 476 & 6,598 \\
Average number of instance per bag & 5.17 & 64.69 \\
Maximal number of instances in a bag & 40 & 1,044 \\
Minimal number of instances in a bag & 2 & 1 \\
\hline
\end{tabular}

Leave-one-out test is performed on each Musk data set. In detail, for $N$ bags, one bag is used to test while the others are used to train a BP-MIP ensemble in a loop of $N$ iterations. In each iteration, bootstrap sampling [3] is used to generate four training sets from the original training set and four versions of BP-MIP neural network are trained respectively on each generated training set. Together with the BP-MIP neural network trained on the original training set, a BP-MIP ensemble containing five versions of BPMIP neural network is constituted. The output of the BP-MIP ensemble under the test bag is determined by the outputs of its component BP-MIP 
neural networks via majority voting. The final predictive accuracy is calculated as the total number of correctly labeled test bags divided by $N$.

Table 2 compares the predictive accuracy of BP-MIP ensemble on the Musk data with those reported in the literatures. Configuration of component BP-MIP neural network is the same as that used in the literature [8].

Table 2. Comparison of the predictive accuracy on the Musk data

\begin{tabular}{lc|lc}
\hline algorithm & $\begin{array}{c}\text { Muskl } \\
\% \text { correct }\end{array}$ & algorithm & $\begin{array}{c}\text { Musk2 } \\
\text { \%correct }\end{array}$ \\
\hline EM-DD & 96.8 & EM-DD & 96.0 \\
Iterated-discrim APR & 92.4 & Iterated-discrim APR & 89.2 \\
Citation- $k$ NN & 92.4 & Relic & 87.3 \\
Diverse Density & 88.9 & Citation- $k$ NN & 86.3 \\
RIPPER-MI & 88.0 & BP-MIP ensemble & $\mathbf{8 4 . 3}$ \\
BP-MIP ensemble & $\mathbf{8 7 . 0}$ & MULTINST & 84.0 \\
BP-MIP & 83.7 & Diverse Density & 82.5 \\
Relic & 83.7 & BP-MIP & 80.4 \\
MULTINST & 76.7 & RIPPER-MI & 77.0 \\
\hline
\end{tabular}

Table 2 shows that BP-MIP ensemble performs better than BP-MIP on both Muskl and Musk2 data sets. Furthermore, the performance of BP-MIP ensemble, i.e. $87.0 \%$ on Muskl and $84.3 \%$ on Musk2, is comparable to $88.9 \%$ on Muskl and $82.5 \%$ on Musk2, i.e. the result achieved by Diverse Density [5], even though the architecture and parameters of component BPMIP neural networks have not been finely tuned.

\section{BP-MIR ENSEMBLE}

In 2001, Amar et al. [1] presented a method for creating artificial multiinstance data. The same as BP-MIP ensemble, leave-one-out test is performed on artificial data sets. The output of the BP-MIR ensemble under the test bag is determined by the outputs of its component BP-MIR neural networks via simple averaging. On the other hand, through rounding the real-valued outputs of component BP-MIR neural networks to 0 or 1 , the predictive error of the BP-MIR ensemble can also be evaluated.

Due to the time limitation, we have only experimented on the data set $\mathrm{LJ}$ 80.166.1. Configuration of component BP-MIR neural network is the same as that used in the literature [7]. Experiments show that, compared with those of BP-MIR, the squared loss of BP-MIR ensemble reduces from 0.0487 to 0.0455 and the predictive error of BP-MIR ensemble reduces from $18.48 \%$ to $11.96 \%$, even though the architecture and parameters of component BP-MIR neural networks have not been finely tuned. 


\section{CONCLUSION}

BP-MIP and BP-MIR are two neural network based multi-instance algorithms designed respectively for classification and regression tasks. In this paper, BP-MIP ensemble and BP-MIR ensemble are constructed correspondingly through employing neural network ensemble techniques. Experiments on benchmark and artificial data sets show that ensembles of multi-instance neural networks are superior to single multi-instance neural networks in solving multi-instance problems.

It is obvious that investigating better configurations of the component neural networks to further improve the generalization ability of BP-MIP ensemble and BP-MIR ensemble is an important issue to be explored in the near future. Furthermore, investigating other ensemble learning techniques to construct ensembles of multi-instance neural networks is another interesting issue for future work.

\section{Acknowledgements}

This work was supported by the National Outstanding Youth Foundation under the Grant No. 60325207 and the National Natural Science Foundation of China under the Grant No. 60105004 .

\section{References}

[1] Amar, R. A., Dooly, D. R., Goldman, D. R. and Zhang, Q.: Multiple-instance learning of real-valued data, In: Proceedings of the 18th International Conference on Machine Learning, pp. 3-10, Williamstown, MA, 2001.

[2] Dietterich, T. G., Lathrop, R. H. and Lozano-Pérez, T.: Solving the multiple-instance problem with axis-parallel rectangles, Artificial Intelligence, 89(1-2) (1997), 31-71.

[3] Efron, B. and Tibshirani, R.: An Introduction to the Bootstrap, Chapman \& Hall, New York, 1993.

[4] Maron, O.: Learning from Ambiguity, Ph.D. thesis, Department of Electrical Engineering and Computer Science, MIT, June 1998.

[5] Maron, O. and Lozano-Pérez, T.: A framework for multiple-instance learning, In: M. I. Jordan and M. J. Kearns, (eds.), Advances in Neural Information Processing Systems 10, pp. 570-576, MIT Press, Cambridge, MA, 1998.

[6] Rumelhart, D. E., Hinton, G. E. and Williams, R. J.: Learning internal representations by error propagation, Nature, 323(9) (1986), 533-536.

[7] Zhang, M.-L. and Zhou, Z.-H.: A neural network based multi-instance regression algorithm, Journal of Software, 14(7) (2003), 1238-1242. (in Chinese)

[8] Zhang, M.-L. and Zhou, Z.-H.: Improve multi-instance neural networks through feature selection, Neural Processing Letters, 19(1) (2004): 1-10.

[9] Zhou, Z.-H. and Zhang, M.-L.: Neural networks for multi-instance learning. Technical Report, AI Lab, Computer Science \& Technology Department, Nanjing University, Nanjing, China, Aug. 2002.

[10] Zhou, Z.-H. and Zhang, M.-L.: Ensembles of multi-instance learners. In: N. Lavrač, D. Gamberger, H. Blockeel and L. Todorovski, (eds.), Lecture Notes in Artificial Intelligence 2837, pp. 492-502, Springer-Verlag, Berlin, 2003. 